\title{
Amplitude Response of an Overload-Resistant Pressure Sensor
}

Kober, Timo

Institut für Elektromechanische Konstruktionen, Technische Universität Darmstadt

Merckstraße 25, 64283 Darmstadt

\begin{abstract}
Differential pressure sensors with silicon diaphragms provide a nominal measuring range of $10 \mathrm{mbar}$ $(1 \mathrm{kPa})$ at a measurement uncertainty of $\leq 2 \cdot 10^{-3}$. An additional external overload protection mechanism is required to achieve an overload reserve up to $160 \mathrm{bar}(16 \mathrm{MPa})$. To integrate the overload protection at the wafer level, a micromechanical overload protection is needed. Such a micromechanical overload protection for differential pressure sensors can be produced from heat-treated glass. The functional principle limits the diaphragm deflection. An overload capability exceeding 50 bar $(5 \mathrm{MPa})$ for a silicon diaphragm with a nominal measuring range of $10 \mathrm{mbar}$ and a diaphragm thickness of $30 \mu \mathrm{m}$ is possible [3]. The dynamic overload capability corresponds to the amplitude response of an overloadresistant pressure sensor. The low pass characteristic is demonstrated by dynamic pressure calibration.
\end{abstract}

\section{Homogenous deflection limiter made of glass}

To prove the concept of homogenous deflection limiters made of glass, a first approach, with one-sided overload protection, has been realized. The important parameters of the production process are time, temperature and wafer thickness [3]. Prior to thermal treatment, the three-layer wafer stack, consisting of two silicon wafers and one glass wafer, must be positioned. The first laser-cut silicon wafer provides a substrate with defined openings (Fig. 1 a).

1

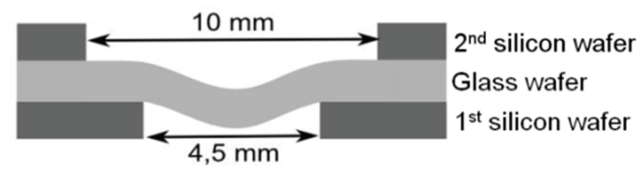

2

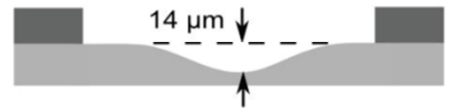

3

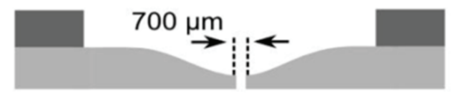

4

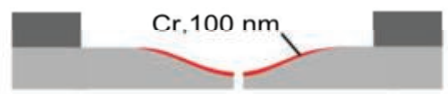

5

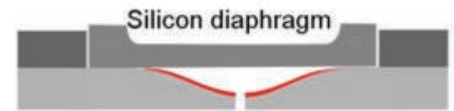

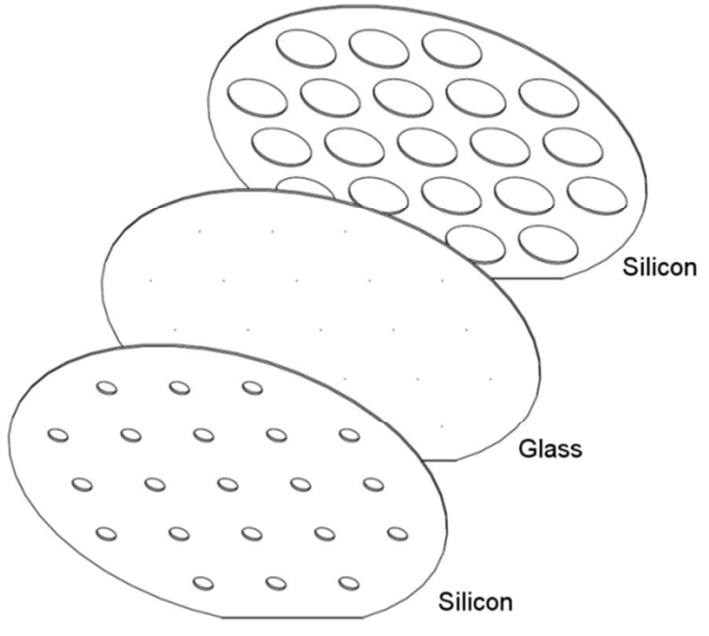

Fig. 1 a) Process steps, b) Wafer level stack [1].

A glass wafer is positioned on this substrate wafer. The placement of the pressure sensor dice is defined by custom openings in the second silicon wafer. Clean room conditions are required to avoid the entrapment of particles. Placing the wafer stack horizontally in a chamber heated up to $750^{\circ} \mathrm{C}$ produces a softening of the glass (Fig. $1 \mathrm{~b}$ ). Because of its own weight, the glass sinks slightly into the laser cut pattern in the silicon substrate wafer [1]. Bonding the silicon wafer and the glass wafer at $750^{\circ} \mathrm{C}$ causes a significant wafer bow. This wafer bow is avoided by using a second silicon wafer on top of the glass wafer. This makes the stack symmetrical (Fig. 1 b).

Further processing requires the removal the first silicon wafer, by lapping or grinding (see numbers 1-5 in Fig. 1 a). Using ultrasonic drilling, a capillary tube with diameter smaller than $700 \mu \mathrm{m}$ is processed into the glass wafer, to ensure pressure equilibrium. Vacuum metalizing of a $100 \mathrm{~nm}$ thin chrome layer is needed to realize selective anodic bonding. Prior to the overload test, a silicon diaphragm is fixated by anodic bonding. Simultaneous processing of 21 pieces is possible. An important property is the automatically flattened surface whose surface roughness is suitable for anodic bonding. Ultrasonic drilling separates the deflection limiters including the silicon diaphragms into round samples with a diameter of $20 \mathrm{~mm}$. 

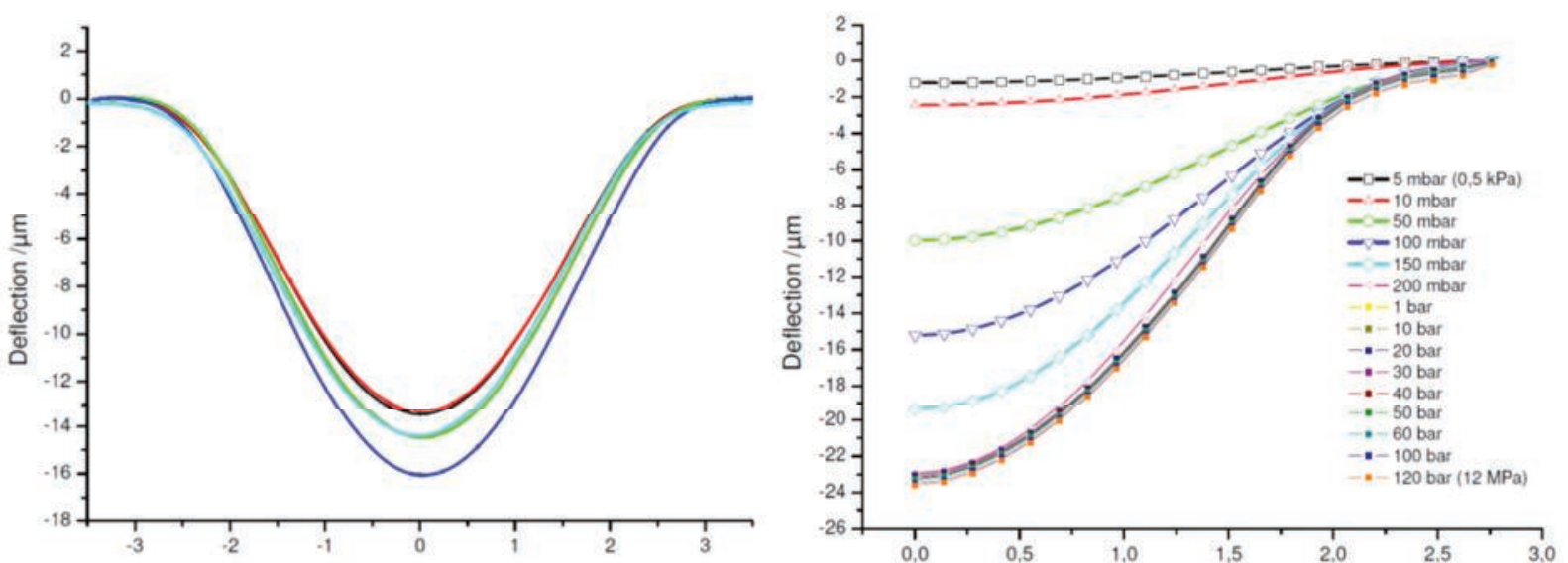

Fig. 2 a) Measured representative curves of 6 glass deflection limiters, b) FEM simulation of diaphragm deflection over radial axis with a real deflection limiter [3].

We have produced deflection limiters whose average depth of the aspheric structure is $14 \mu \mathrm{m}$ (Fig. $2 \mathrm{a}$ ). The defection of a virtual diaphragm is simulated on a real deflection limiter with depth of $24 \mu \mathrm{m}$ (Fig. $2 \mathrm{~b}$ ). The simulation shows a free diaphragm movement for the nominal pressure range of 10 mbar. For differential pressure $\Delta p>200$ mbar, the silicon diaphragm adapts the deflection limiters aspheric shape. If the overload pressure increases, the deflection is still limited to $24 \mu \mathrm{m}$ and the structured deflection limiter provides an adapted, aspheric shape which prevents the diaphragm destruction.

\section{Dynamic pressure source - mechanical design and concept}

For a nominal measuring range of $\Delta p_{N}=10 \mathrm{mbar}$, the capacitive or piezoresistive principle is used for differential pressure measurement, as well as the resonance analysis of thin silicon beams. In all cases a $15 \mu \mathrm{m}-30 \mu \mathrm{m}$ thin silicon diaphragm is used as deformation body in order to detect the differential

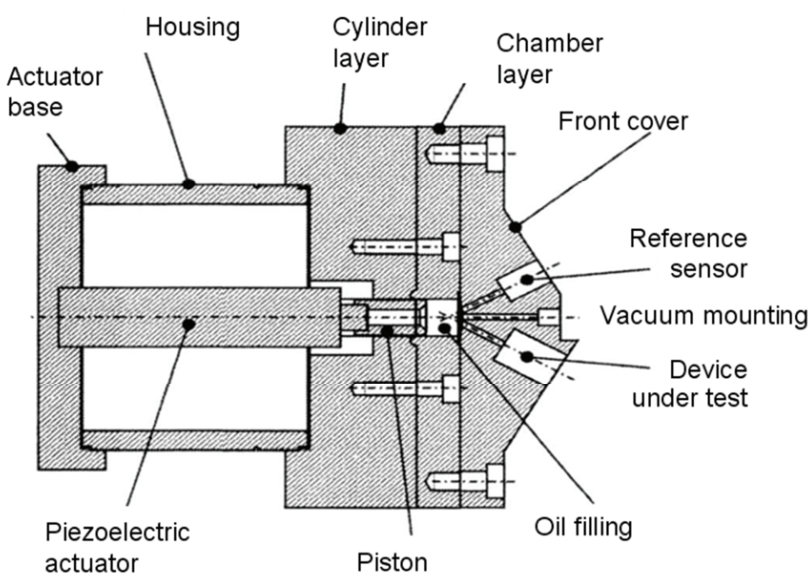

Fig. 3 Pressure source, mechanical design [2]. pressure. The dynamic amplitude and phase response changes with extended capillary tubes or static pressure overload protection diaphragms. Usually, the upper cut-off frequency of silicon pressure sensors without housing and overload protection is in the range of $10 \mathrm{kHz}-$ $100 \mathrm{kHz}$ depending on the pressure range the sensor is designed for. Sensor packaging in general affects the nominal frequency range [2]. This also applies to other physical working principles, like thin film or ceramic pressure sensors. The influence of packaging and overload protection on the sensors frequency response has long been object of research at our department. To be able to prove theoretical results, we built a dynamic pressure source whose design, specific values and applications will be discussed below. The dynamic pressure source consists of a network analyzer and a piezoelectric stack actuator, which is driven by a high voltage amplifier up to $-1000 \mathrm{~V}$. The actuator is used as a force source connected to a piston and a steel diaphragm (Fig. 3). The pressure $p$ in the hermetically sealed chamber layer increases proportionally for small diaphragm deflections. A properly deaerated oil filled chamber is crucial to achieve the maximal pressure amplitude. With every new measurement setup a vacuum pump is connected to the front cover to deaerate the oil filled chamber, because dissolved gas in the Table 1 Dynamic pressure source, typical values. oil

\begin{tabular}{|c|c|c|}
\hline Frequency range & $1-10.000$ & $\mathrm{~Hz}$ \\
\hline Amplitude & 15 & $\mathrm{mbar}$ \\
\hline Amplitude maximum & 700 & $\mathrm{mbar}$ \\
\hline Amplitude uncertainty & $<0,1$ & $\mathrm{~dB}$ \\
\hline Operating temperature & 20 & ${ }^{\circ} \mathrm{C}$ \\
\hline Reference sensor & $701 \mathrm{~A}$ & Kistler \\
\hline Reference sensor linearity & 0,5 & $\%$ FSO \\
\hline
\end{tabular}


filling considerably increases the acoustic compliance. A network analysis for harmonic pressure excitation is possible. A piezoelectric pressure sensor is used as a reference to detect the generated harmonic pressure amplitude in a wide frequency range from quasi static behavior up to $10 \mathrm{kHz}$ (Table 1). A charge amplifier provides the needed reference voltage to control the generated pressure amplitude to a user defined level. The device under test and the reference sensor are mounted at the same front cover of the chamber layer. The pressure in the unregulated operation mode is limited by the allowed maximal actuator excitation $\xi_{\max }<60 \mu \mathrm{m}$. The pressure amplitude and phase response of the dynamic pressure source typically follows a low pass characteristic with resonance at the actuator resonance frequency. The signal of the reference sensor and the device under test are compared as long as the pressure source generates a detectable pressure.

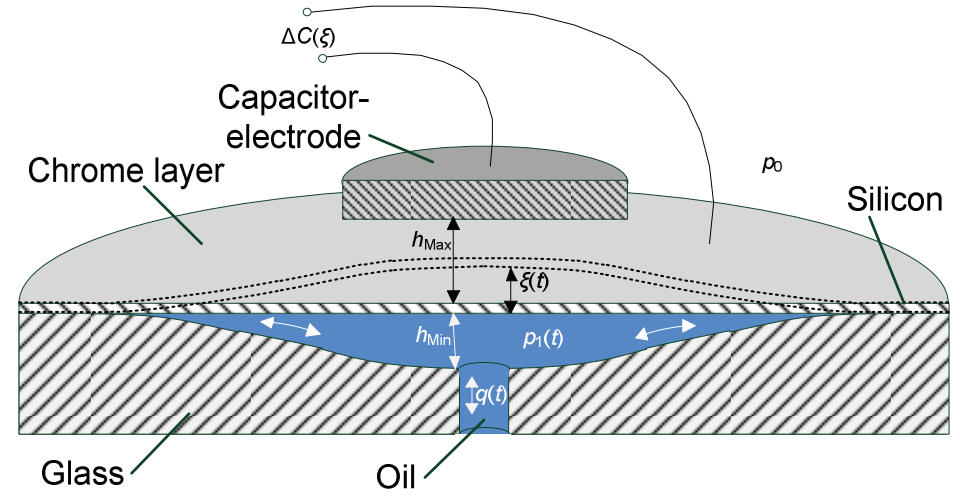

Fig. 4 Scheme of oil filled pressure sensor.

\section{Measuring the Frequency dependent diaphragm deflection}

The current sensor design provides the MEMS-Device with an external capacitive device.

The overload resistant pressure sensor is filled up one sided with silicone oil as a transfer fluid (Fig. 4). A capillary tube $(\varnothing=500 \mu \mathrm{m})$ connects the pressure sensor with the introduced dynamic pressure source. The piston deflection causes a volume flow which builds up a pressure drop $\Delta p=p_{1}-p_{0}$ over the diaphragm. As a consequence a deflection of the Silicon diaphragm occurs, which is measured with a deflection sensor (Table 2). The reference pressure is detected by a piezoresistive pressure sensor. The capacitor consists of a metal electrode and a vacuum deposited chrome layer on top of the silicon diaphragm. The chrome layer has a galvanic contact to the capacitive device. A distance of $h_{\mathrm{Max}}=20 \mu \mathrm{m}$

Table 2 Capacitive deflection measurement, technical data.

\begin{tabular}{|c|c|c|}
\hline Frequency response & 2 & $\mathrm{kHz}$ \\
\hline Measuring range & 200 & $\mu \mathrm{m}$ \\
\hline Linearity & 0,1 & $\% \mathrm{FSO}$ \\
\hline Capacitive device & Micro-Epsilon & $\mathrm{CS02}$ \\
\hline Reference pressure & $\mathrm{MSI} 5108 \mathrm{C}$ & $15 \mathrm{PSI}$ \\
\hline
\end{tabular}

assures free movement of the diaphragm. Ambient air fills up the electrode spacing and is used as a dielectric medium. The aspheric structure provides a depth of $h_{\mathrm{Min}}=18 \mu \mathrm{m}$.

A peak deflection of $\xi_{\max }=10 \mu \mathrm{m}$ at $\mathrm{a}$ diaphragm diameter of $6 \mathrm{~mm}$ causes an arc radius of about $110 \mathrm{~mm}$. This systematic error due to non-planar surfaces has not been taken

into account. The effective measurement area of about $\varnothing=2 \mathrm{~mm}$ is adequate to detect the diaphragm deflection. To achieve a first impression of the dynamic behavior, a step response was sampled. At the beginning of the measurement a low pressure $p_{1}<<p_{0}$ pulls the diaphragm down on the deflection limiter (Fig. 5). At $t=5 \mathrm{~s}$ a differential pressure $p_{1}-p_{0}=$ $30 \mathrm{mbar}$ is connected to the capillary system. In dependence of time, the constant pressure of $\Delta p=$ 30 mbar causes a volume flow and the diaphragm lifts of the deflection limiter. At $t=25 \mathrm{~s}$ the equilibrium state is reached.

The deflection velocity dependents on the oil gap between diaphragm and deflection limiter. For harmonic pressure excitation a deflection in the range of $\xi_{\operatorname{Max}}=10 \mu \mathrm{m}$ is preferred, to achieve a solid and detectable signal, while proportional conditions are observed (Fig. 6).

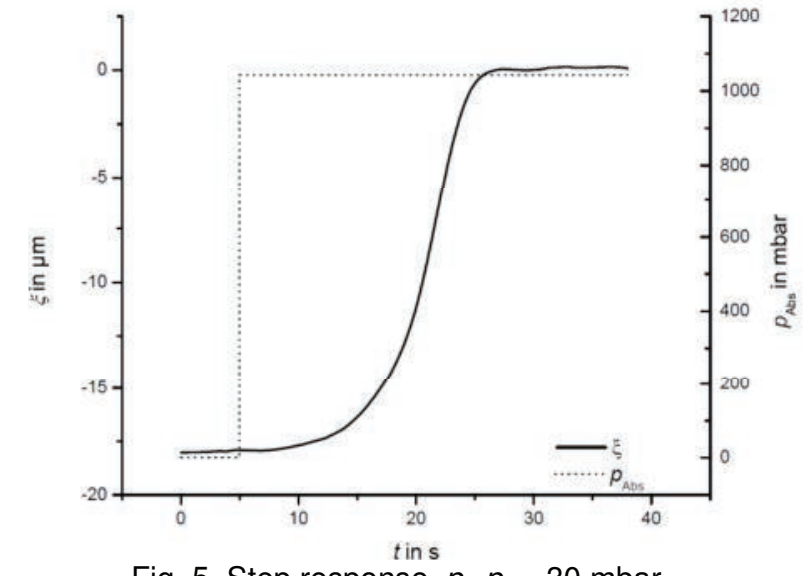

Fig. 5. Step response, $p_{1}-p_{0}=30$ mbar. 

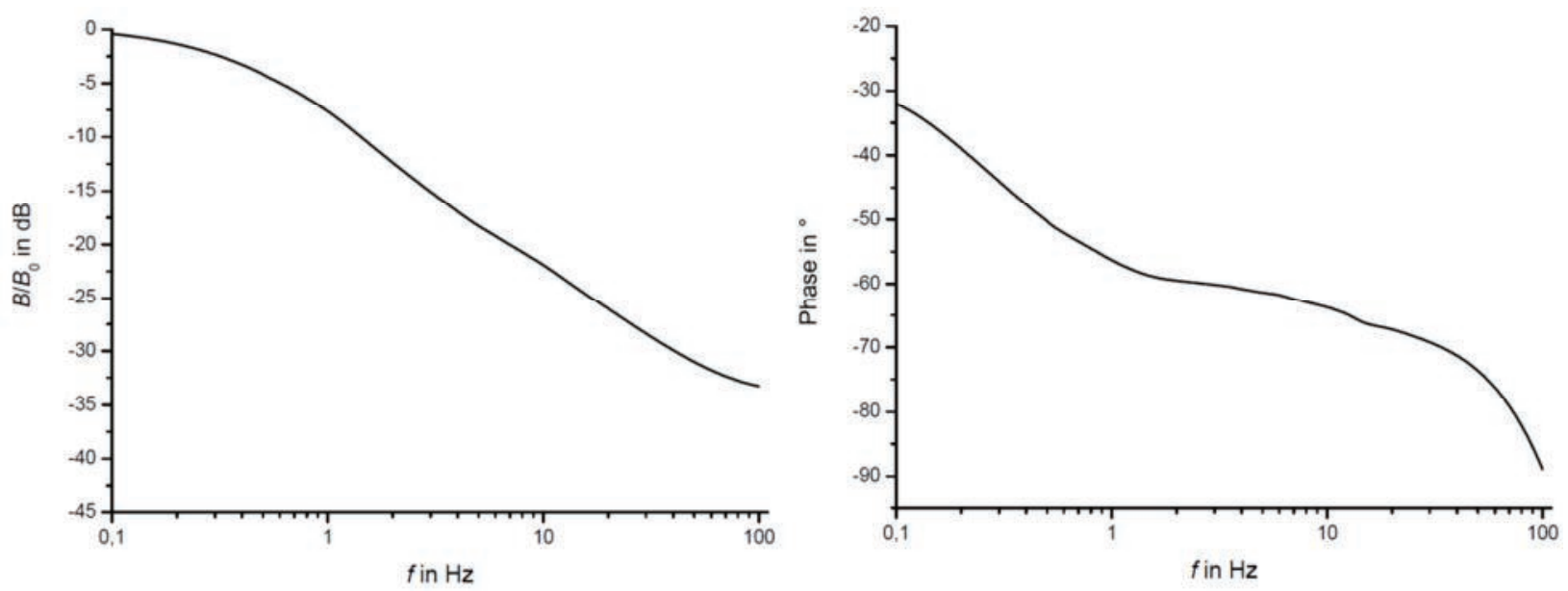

Fig. 6 Overload resistant pressure sensor $\xi / \underline{p}_{1}$ a) Amplitude response, b) Phase response.

Pressure excitation results in a harmonic diaphragm deflection in the linear operating range. Excitation with a dynamic pressure of $\hat{\underline{p}}=60 \mathrm{mbar}$ results in a diaphragm deflection of $\hat{\xi}=7 \mu \mathrm{m}$ at $f=0,1 \mathrm{~Hz}$. For small amplitudes, the pressure drop over the diaphragm is proportional to the diaphragm deflection. Considering the electrical amplification factors equation (1) corresponds to figure 6 a).

$$
B_{0}=\underline{\hat{u}}_{2}(\underline{\xi}) / \underline{\hat{u}}_{1}(\underline{p})
$$

The amplitude response of the overload resistant pressure sensor shows a strong low pass characteristic. The influence of the oil viscosity, oil temperature and capillary design are current fields of our research.

\section{Conclusion}

The overload resistant pressure sensor provides a static overload capability exceeding 50 bar. It is assumed that the strong low pass characteristic $f_{0}<1 \mathrm{~Hz}$ leads also to an overload protection for dynamic pressure excitation. Especially pressure transients, caused by valve switching, could be damped. This sensor concept will be developed to a symmetrical layout with double-sided overload protection. Depending on the capillary diameter, the dynamic overload capability corresponds to the frequency response of the silicon diaphragm and the acoustic system.

\section{References}

[1] Kober T, Werthschützky R, Mikromechanischer Überlastschutz für Drucksensoren durch strukturierte Gegenlager aus Glas. In: Sensoren und Messsysteme 2010, Berlin: VDE Verlag GmbH, ISBN 978-3-8007-3260-9; 2010.

[2] Kober T, Sindlinger S, Werthschützky R, Analyse des Übertragungsverhaltens von Differenzdrucksensoren durch dynamische Druckkalibrierung. In: Technisches Messen, München: Oldenbourg Verlag, ISSN 0171-8096; 2010, p. 83-88.

[3] Kober T, Werthschützky R, Overload-resistant pressure sensors in the nominal range of $10 \mathrm{mbar}(1 \mathrm{kPa})$. In: Eurosensor XXIV Conference, September 5-8, 2010, Linz, Austria. Proc. Eurosensors XXIV, Konferenz- oder Workshop-Beitrag, (2010) 